IMA Journal of Applied Mathematics (2013) Page 1 of 11

doi:10.1093/imamat/xxx000

\title{
Analysis of the equations governing the motion of a degrading elastic solid due to diffusion of a fluid
}

\author{
R. QUINTANILLA* \\ Matematica Aplicada 2, U.P.C., Colom, 11, 08222 Terrassa, Barcelona, Spain \\ *Corresponding author: Ramon.Quintanilla@upc.edu \\ AND \\ K. R. RAJAGOPAL \\ Mechanical Engineering, Texas A \& M University, College Station, TX 77843-3123, USA
}

[Received on 19 November 2013]

\begin{abstract}
Degradation of solids bearing load due to the infusion of moisture when exposed to the environment can leads to a decrease in their load carrying capacity and can also lead to the failure of the body from performing its intended task. In this short paper, we study some qualitative properties of the solution to systems of equations that describe the degradation in a linearized elastic solid due to the diffusion of a fluid. The model that is considered allows for the material properties of the solid to depend on the concentration of the diffusing fluid. While the load carrying capacity of a solid could decrease or increase due to the infusion of a fluid, we consider the case when degradation takes place. We are able to obtain results concerning the uniqueness of solutions to the problem under consideration. We also consider special anti-plane and quasi-static deformations of the body.
\end{abstract}

Keywords: Degradation, Diffusion, Constitutive relations, Uniqueness, Ani-plane strain.

2010 Math Subject Classification: 74F10, 74H25.

\section{Introduction}

The ability of a body to withstand loads decreases or increases due to a variety of reasons: changes in the temperature of the body, infusion of a fluid and subsequent chemical reactions, electromagnetic radiation, aging, etc. For instance, the diffusion of moisture in materials like concrete and many metals is the primary cause of degradation of such materials when exposed to the natural elements. Several models have been introduced to describe the damage and thus the reduced load carrying capacity due to the influence of hostile environmental conditions (it is also possible that the load carrying capacity of certain bodies might increase due to the healing that is effected by the influence of chemicals), but most of them have been ad hoc empirical models. For instance, in problems concerning the diffusion of fluids through solids and the subsequent degradation of the solids, in most studies the variation of the concentration of the fluids is assumed a priori and the convection diffusion problem is not solved (see Bouadi \& Sun (1989,1990), Snead and Palazotto (1983), Yen \& Yen (1989), Kardomateas \& Chung (1993). At the other extreme, Weistman (1987a,1987b) has developed a very general framework in which he introduces a damage tensor and the specific Helmholtz potential is assumed to depend on this damage tensor in addition to several other variables. Unfortunately, the general theory involves material moduli that depend, in the case of transversely isotropic solids on as many as thirty two invariants, and is thus totally unusable as one cannot put into place an experimental program within which one could 
measure material functions of thirty two variables.

Recently, Muliana et al.(2009) adopted a somewhat different approach to study the reduction in load carrying capacity of solids, due to the diffusion of a fluid. They assumed that the material moduli of the solid depend on the concentration of the fluid. Using such an approach, Muliana et al.(2009) studied the degradation of an elastic composite cylinder due to the diffusion of a fluid, by assuming that the value of the material moduli decrease with increasing fluid content. They found that significant changes take place over time with regard to the stress and strains within the body. For instance, the bodies "stress-relax" due to the infusion of the fluid, but this stress-relaxation is different from that undergone by viscoelastic solids. Similar stress-relaxation has been observed in solids that age (see Rajagopal \& Wineman (2004)).

Unsteady motions of degrading and aging elastic cylinders have been studied recently by Darbha \& Rajagopal (2009). Soares et al.(2010) introduce a damage parameter, an internal variable, that depends on the strain as well as the fluid concentration, to study the response of biodegradable polymeric stents. In all these studies, the problem is simplified to that being governed by the balance of linear momentum and a convection-diffusion equation for the motion of the fluid. This is a tremendous simplification of the full problem, but such a simplification might yield some insight into the problem under consideration.

A more thorough and comprehensive study of the problem of degradation of the solid due to the diffusion of a fluid could be achieved by resorting to the use of the theory of mixtures (theory of interacting continua) that traces its roots to the seminal work of Fick (1855) and Darcy (1856). The theory was given a firm mathematical footing by Truesdell $(1957 \mathrm{a}, 1957 \mathrm{~b}, 1984)$ and a review of the same can be found in the articles by Atkin \& Craine (1976), Bowen (1976) and the books by Samohyl (1987) and Rajagopal \& Tao (1995). The basic idea behind mixture theory is that various constituents co-occupy the region of the mixture, in a homogenized sense. Each constituents has its own kinematics and basic balance laws are specified for each constituents. However, the theory is not without its own problems. There are inherent difficulties concerning the specification of initial and boundary conditions within the theory (see Rajagopal \& Tao (1995)). In any event, the theory is a lot more complicated to use than the simplified approach adopted by Muliana et al. (2009), and this particular study concerns certain mathematical results concerning the latter approach.

An interesting feature concerning the degradation of solids due to the diffusion of a fluid arises from the fact that the diffusion can progress differently along different directions. The anisotropy with regards to the diffusion might or might not correlate with the anisotropy of the solid. In this paper, we shall not consider such possibilities though a simple modification to the governing equations can address this issue. We are interested in the diffusion of a fluid through an isotropic linerized elastic solid, and we shall assume that the Lamé constants depend on the concentration. It is possible that the value of the Lamé constants increase or decrease with the concentration. Enhancement of properties are relevant to both biomedical and civil engineering applications. However, most often the body's load carrying capacity diminishes due to the diffusion of the fluid, the common mechanism for degradation is oxidation.

We also account for the possibility that the diffusivity changes due to the swelling of the solid due to the absorption of the fluid. This physical phenomenon has important mathematical consequences; it couples the reaction-diffusion equations to the balance of the linear momentum.

The plan of this paper is as follows: Section 2 is devoted to the documentation of the basic equations that we are going to work with. In Section 3 we investigate the uniqueness of solutions for the dynamical problem. The main argument is the use of the energy methods. In Section 4 we show how the problem is much easier when we restrict our attention to anti-plane shear deformations. Some possible results are discussed, but without any proof. In Section 5, we consider the case of quasi-static deformations 
and we prove that the uniqueness of solutions fails in the particular case when the concentration is 1 everywhere and when the material moduli satisfy certain conditions. However, we can not expect that this result will hold in the general case, that is when the special condition concerning the material moduli is not satisfied. In fact, we prove the uniqueness of solutions in the case of anti-plane shear deformations.

\section{Basic Equations}

The equations governing the evolution of a degrading linearized elastic body subject to the diffusion of a fluid are:

$$
\begin{gathered}
\rho u_{i, t t}=T_{i j, j}, \\
c_{t}+\left(c \dot{u}_{i}\right)_{, i}=\left(D\left(\varepsilon_{k l}\right) c_{, i}\right)_{, i},
\end{gathered}
$$

where the constitutive equation for the stress is:

$$
T_{i j}=(\lambda(c)+\mu(c)) \delta_{i j} u_{k, k}+\mu(c) u_{i, j}
$$

We recall that $u_{i}$ is the displacement vector, $\varepsilon_{k l}$ is the linearized strain tensor given through $\varepsilon_{k l}=$ $\left(u_{k, l}+u_{l, k}\right) / 2, c$ is the concentration which satisfies $0<c \leqslant 1, D\left(\varepsilon_{k l}\right)$ denotes the strain dependent diffusivity and $\lambda$ and $\mu$ are the concentration dependent Lamé coefficients. When we consider quasistatic deformations, we assume that the motion is such that we can neglect the acceleration components and then the equation (2.1) becomes

$$
T_{i j, j}=0
$$

In this paper we study several qualitative properties of the solutions of the system (2.1)-(2.3) or (2.2)-(2.4). To this end, it will be convenient to set down the initial and boundary conditions we are going to work with as well as the assumptions that we shall make concerning the constitutive functions.

The functions $\lambda, \mu$ are defined in the following manner

$$
\lambda(c)=\lambda_{0}-\lambda_{1} c, \mu(c)=\mu_{0}-\mu_{1} c,
$$

where $\lambda_{0}, \lambda_{1}, \mu_{0}$ and $\mu_{1}$ are constants. In much of the article, we are going to assume that

$$
\lambda_{0}>\lambda_{1}, \mu_{0}>\mu_{1} .
$$

It is worth noting that for functions of the form $\mu=\mu_{0} \exp \left(-\gamma_{1} c\right), \lambda=\lambda_{0} \exp \left(-\gamma_{2} c\right)$ one can carry out an analysis similar to that presented below.

With respect the function $D$ we assume that it is a function of the linearized strain and must satisfy the following two conditions:

(i) There exists a positive constant $d_{0}$ such that

$$
D\left(\varepsilon_{k l}\right) \xi_{i} \xi_{i} \geqslant d_{0} \xi_{i} \xi_{i}
$$

for every vector $\left(\xi_{i}\right)$ and for every $\varepsilon_{k l}$.

(ii) The function $D$ is such that there exists a continuous function $K(.,$.$) , such that$

$$
\left|D\left(\varepsilon_{k l}^{(1)}\right)-D\left(\varepsilon_{k l}^{(2)}\right)\right| \leqslant K\left(\varepsilon_{i j}^{(1)}, \varepsilon_{i j}^{(2)}\right)\left|\varepsilon_{k l}^{(1)}-\varepsilon_{k l}^{(2)}\right|
$$

for every $\varepsilon_{k l}^{(i)}, i=1,2$. 
To render the problem determinate we will impose boundary conditions for the displacement and the concentration

$$
u_{i}(\mathbf{x}, t)=u_{i}^{\partial}(\mathbf{x}, t), \quad c(\mathbf{x}, t)=c^{\partial}(\mathbf{x}, t), \quad \mathbf{x} \in \partial B, 0<t \leqslant t_{0}<\infty,
$$

and initial conditions

$$
u_{i}(\mathbf{x}, 0)=u_{i}^{0}(\mathbf{x}), \quad \dot{u}_{i}(\mathbf{x}, 0)=v_{i}^{0}(\mathbf{x}), \quad c(\mathbf{x}, 0)=c^{0}(\mathbf{x}), \mathbf{x} \in \partial B .
$$

It is worth noting that these initial and boundary conditions must be assumed when we consider the dynamic problem, however when we consider the quasi-static problem, we only assume the third condition in (2.10).

We can also consider the class of incompressible linearized elastic solids. The constitutive relation for such bodies takes the form

$$
\mathbf{T}=-p \mathbf{I}+2 \mu(c) \boldsymbol{\varepsilon}
$$

where $-p \mathbf{I}$ is the indeterminate spherical stress due to the constraint of the incompressibility. In this case the governing equations reduces to

$$
\begin{gathered}
\left(\mu(c) u_{i, j}\right)_{, j}+p_{, i}=f_{i}(\mathbf{x}, t), u_{i, i}=0, \\
c_{t}+\left(c \dot{u}_{i}\right)_{, i}=\left(D\left(\varepsilon_{k l}\right) c_{, i}\right)_{, i}+g(\mathbf{x}, t) .
\end{gathered}
$$

Anti-plane shear deformations are one of the easiest deformations to study within the context of elasticity. We shall study such anti-plane shear deformations in the case of the degradation of an elastic cylinder due to the diffusion of a fluid. In this case we look for solutions of the form

$$
u_{1}(\mathbf{x}, t)=u_{2}(\mathbf{x}, t)=0, u_{3}(\mathbf{x}, t)=u\left(x_{1}, x_{2}, t\right), c(\mathbf{x}, t)=c\left(x_{1}, x_{2}, t\right) .
$$

Here $\left(x_{1}, x_{2}\right)$ lies in a two dimensional domain $S$. The functions $(u, c)$ must satisfy the system

$$
\rho u_{, t t}=\left(\left(\mu_{0}-\mu_{1}\left(c\left(x_{1}, x_{2}, t\right)\right) u_{, \alpha}\right)_{, \alpha} c_{, t}=\left(D\left(u_{, 1}, u_{, 2}\right) c_{, \alpha}\right)_{, \alpha}\right.
$$

where $\alpha=1,2$. To define the problem, we need to impose initial conditions

$$
u\left(x_{1}, x_{2}, 0\right)=u^{0}\left(x_{1}, x_{2}\right), v\left(x_{1}, x_{2}, 0\right)=v^{0}\left(x_{1}, x_{2}\right), c\left(x_{1}, x_{2}, 0\right)=c^{0}\left(x_{1}, x_{2}\right),\left(x_{1}, x_{2}\right) \in S,
$$

and the boundary conditions

$$
u\left(x_{1}, x_{2}, t\right)=u^{\partial}\left(x_{1}, x_{2}, t\right), c\left(x_{1}, x_{2}, t\right)=c^{\partial}\left(x_{1}, x_{2}, t\right),\left(x_{1}, x_{2}\right) \in \partial S, 0<t \leqslant t_{0}<\infty .
$$

When $\mu=\mu_{0} \exp \left(-\gamma_{1} c\right)$ the equation (2.14) becomes $\rho u_{, t t}=\left(\mu_{0} \exp \left(-\gamma_{1} c\right) u_{, \alpha}\right)_{, \alpha} \cdot$,

In the case of quasi-static deformations the first of the equations in (2.14) becomes

$$
\left(\left(\mu_{0}-\mu_{1}\left(c\left(x_{1}, x_{2}, t\right)\right) u_{, \alpha}\right)_{, \alpha}=0 .\right.
$$

To complete this problem, we adjoin the boundary conditions as in (2.16) and only the third condition in (2.15).

When $\mu=\mu_{0} \exp \left(-\gamma_{1} c\right)$ the equation (2.17) becomes

$$
\left(\mu_{0} \exp \left(-\gamma_{1} c\right) u_{, \alpha}\right)_{, \alpha}=0 .
$$




\section{Uniqueness of solutions}

In this section we will prove the uniqueness of solutions for the problem determined by the system (2.1)-(2.3), when we assume the conditions (2.5), (2.6), (2.7), (2.8), the boundary conditions (2.9) and the initial conditions (2.10).

We assume the existence of two solutions $\left(u_{i}^{(j)}, c^{(j)}\right), j=1,2$ such that they satisfy the problem and such that $c^{(j)}$ satisfies $0<c^{(j)} \leqslant 1$ for $j=1,2$. It is worth noting that this condition is imposed to guarantee that the solutions are physically meaningful.

In this section we will assume that

$$
u_{i}^{(j)} \in C^{2,0} \cap C^{0,1}, c^{(j)} \in C^{0,1}, j=1,2 .
$$

It is worth noting that $u_{i, k l}^{(j)}, \dot{u}_{i}^{(j)}$ and $\dot{c}^{(j)}$ are bounded on $B$ for every $0 \leqslant t \leqslant t_{0}<\infty$ and for every $j=1,2$.

We define

$$
w_{i}=u_{i}^{(1)}-u_{i}^{(2)}, h=c^{(1)}-c^{(2)} .
$$

The functions $\left(w_{i}, h\right)$ satisfy the problem determined by the system

$$
\begin{gathered}
\rho w_{i, t t}=\left(\left(\lambda\left(c^{(1)}\right)+\mu\left(c^{(1)}\right)\right) \delta_{i j} u_{k, k}^{(1)}+\mu\left(c^{(1)}\right) u_{i, j}^{(1)}\right)_{, j} \\
-\left(\left(\lambda\left(c^{(2)}\right)+\mu\left(c^{(2)}\right)\right) \delta_{i j} u_{k, k}^{(2)}+\mu\left(c^{(2)}\right) u_{i, j}^{(2)}\right)_{, j} \\
h_{, t}+\left(c^{(1)} \dot{u}_{i}^{(1)}\right)_{, i}-\left(c^{(2)} \dot{u}_{i}^{(2)}\right)_{, i}=\left(D\left(\varepsilon_{k l}^{(1)}\right) c_{, i}^{(1)}\right)_{, i}-\left(D\left(\varepsilon_{k l}^{(2)}\right) c_{, i}^{(2)}\right)_{, i}
\end{gathered}
$$

the initial conditions

$$
w_{i}(\mathbf{x}, 0)=\dot{w}_{i}(\mathbf{x}, 0)=h(\mathbf{x}, 0)=0 \quad \mathbf{x} \in B
$$

and the boundary conditions

$$
w_{i}(\mathbf{x}, t)=h(\mathbf{x}, t)=0 \quad(\mathbf{x}, t) \in \partial B \times\left(0, t_{0}\right) .
$$

The system can be written as

$$
\begin{gathered}
\rho w_{i, t t}=\left(\left(\lambda\left(c^{(1)}\right)+\mu\left(c^{(1)}\right)\right) \delta_{i j} w_{k, k}+\mu\left(c^{(1)}\right) w_{i, j}\right)_{, j}-\left(\left(\lambda_{1}+\mu_{1}\right) h \delta_{i j} u_{k, k}^{(2)}+\mu_{1} h u_{i, j}^{(2)}\right)_{, j} \\
h_{, t}+\left(h \dot{u}_{i}^{(1)}\right)_{, i}+\left(c^{(2)} \dot{w}_{i}\right)_{, i}=\left(D\left(\varepsilon_{k l}^{(1)}\right) h_{, i}\right)_{, i}+\left(\left(D\left(\varepsilon_{k l}^{(1)}\right)-D\left(\varepsilon_{k l}^{(2)}\right)\right) c_{, i}^{(2)}\right)_{, i} .
\end{gathered}
$$

If we define the function

$$
E(t)=\frac{1}{2} \int_{B}\left(\rho \dot{w}_{i} \dot{w}_{i}+\mu\left(c^{(1)}\right) w_{i, j} w_{i, j}+\left(\lambda\left(c^{(1)}\right)+\mu\left(c^{(1)}\right)\right) w_{i, i} w_{j, j}+h^{2}\right) d v .
$$

We have

$$
\begin{aligned}
\frac{d E}{d t}= & -\frac{\mu_{1}}{2} \int_{B} \dot{c}^{(1)} w_{i, j} w_{i, j} d v-\frac{\lambda_{1}+\mu_{1}}{2} \int_{B} \dot{c}^{(1)} w_{i, i} w_{j, j} d v \\
& +\left(\lambda_{1}+\mu_{1}\right)\left(\int_{B} \dot{w}_{i} h_{, i} u_{k, k}^{(2)} d v+\int_{B} \dot{w}_{i} h u_{k, k i}^{(2)} d v\right)
\end{aligned}
$$


6 of 11

R.QUINTANILLA AND K.R.RAJAGOPAL

$$
\begin{gathered}
+\mu_{1}\left(\int_{B} \dot{w}_{i} h_{, j} u_{i, j}^{(2)} d v+\int_{B} \dot{w}_{i} h u_{i, j j}^{(2)} d v\right)+\int_{B}\left(h h_{, i} \dot{u}_{i}^{(1)}+c^{(2)} h_{, i} \dot{w}_{i}\right) d v \\
-\int_{B} D\left(\varepsilon_{k l}^{(1)}\right) h_{, i} h_{, i} d v-\int_{B}\left(D\left(\varepsilon_{k l}^{(1)}\right)-D\left(\varepsilon_{k l}^{(2)}\right)\right) h_{, i} c_{, i}^{(2)} d v
\end{gathered}
$$

In view of continuity we have the existence of several positive constants $C_{i}$ such that

$$
\begin{gathered}
\left|-\frac{\mu_{1}}{2} \int_{B} \dot{c}^{(1)} w_{i, j} w_{i, j} d v\right| \leqslant C_{1} \int_{B} \mu\left(c^{(1)}\right) w_{i, j} w_{i, j} d v \\
\left|\frac{\lambda_{1}+\mu_{1}}{2} \int_{B} \dot{c}^{(1)} w_{i, i} w_{j, j} d v\right| \leqslant C_{2} \int_{B}\left(\lambda\left(c^{(1)}\right)+\mu\left(c^{(1)}\right)\right) w_{i, i} w_{j, j} d v \\
\left|\left(\lambda_{1}+\mu_{1}\right) \int_{B} \dot{w}_{i} h_{, i} u_{k, k}^{(2)} d v\right| \leqslant C_{3} \int_{B} \rho \dot{w}_{i} \dot{w}_{i} d v+\delta_{1} \int_{B} D h_{, i} h_{, i} d v \\
\left|\left(\lambda_{1}+\mu_{1}\right) \int_{B} \dot{w}_{i} h u_{k, k i}^{(2)} d v\right| \leqslant C_{4}\left(\int_{B} \rho \dot{w}_{i} \dot{w}_{i} d v+\int_{B} h^{2} d v\right) \\
\left|\mu_{1} \int_{B} \dot{w}_{i} h_{, j} u_{i, j}^{(2)} d v\right| \leqslant C_{5} \int_{B} \rho \dot{w}_{i} \dot{w}_{i} d v+\delta_{2} \int_{B} D\left(\varepsilon_{k l}^{(1)}\right) h_{, i} h_{, i} d v \\
\left|\mu_{1} \int_{B} \dot{w}_{i} h u_{i, j j}^{(2)} d v\right| \leqslant C_{6}\left(\int_{B} \rho \dot{w}_{i} \dot{w}_{i} d v+\int_{B} h^{2} d v\right) \\
\left|\int_{B}\left(h h_{, i} \dot{u}_{i}^{(1)}+c^{(2)} h_{, i} \dot{w}_{i}\right) d v\right| \leqslant C_{7}\left(\int_{B}\left(\dot{\rho} w_{i} \dot{w}_{i}+h^{2}\right) d v\right)+\delta_{3} \int_{B} D\left(\varepsilon_{k l}^{(1)}\right) h_{, i} h_{, i} d v .
\end{gathered}
$$

We note that the constants $\delta_{i}, i=1,2,3$ are positive constants than can be picked as small as we want it to be. Again, continuity and the condition (2.8) for the function $D$ yield

$$
\left|\int_{B}\left(D\left(\varepsilon_{k l}^{(1)}\right)-D\left(\varepsilon_{k l}^{(2)}\right)\right) h_{, i} c_{, i}^{(2)} d v\right| \leqslant C_{8} \int_{B} \mu\left(c^{(1)}\right) w_{i, j} w_{i, j} d v+\delta_{4} \int_{B} D\left(\varepsilon_{k l}^{(1)}\right) h_{, i} h_{, i} d v,
$$

where $\delta_{4}$ can be picked to be as small as we want and $C_{8}$ is a positive constant. Thus, we obtain

$$
\begin{gathered}
\frac{d E}{d t} \leqslant K_{1} \int_{B}\left(\rho \dot{w}_{i} \dot{w}_{i}+\mu\left(c^{(1)}\right) w_{i, j} w_{i, j}+\left(\lambda\left(c^{(1)}\right)+\mu\left(c^{(1)}\right)\right) w_{i, i} w_{j, j}+h^{2}\right) d v \\
+\delta \int_{B} D\left(\varepsilon_{k l}^{(1)}\right) h_{, j} h_{, j} d v-\int_{B} D\left(\varepsilon_{k l}^{(1)}\right) h_{, i} h_{, i} d v
\end{gathered}
$$

where $\delta$ is a positive constant that can be picked to be as small as we want and $K_{1}$ is a constant which depends on the $C_{i}, i=1 \ldots 8$

We know that we can take $\delta$ less than unity and then, we obtain that there exists a positive constant $K_{2}$ such that

This inequality implies that

$$
\frac{d E}{d t} \leqslant K_{2} E(t), t \in\left[0, t_{0}\right]
$$

$$
E(t) \leqslant E(0) \exp \left(K_{2} t\right), t \in\left[0, t_{0}\right] .
$$

As we assume that the initial conditions satisfy (3.5), we see that $E(0)=0$ and then $E(t)=0$ for every $t \in\left[0, t_{0}\right]$. Thus we have proved the following result. 
THEOREM 3.1 . Let $\left(u_{i}^{(j)}, c^{(j)}\right), j=1,2$ be two solutions satisfying condition (3.1) of the problem determined by the system (2.1)-(2.3), when we assume the conditions (2.5), (2.6), (2.7), (2.8), the boundary conditions (2.9) and the initial conditions (2.10) for every $t \in\left(0, t_{0}\right)$ where $t_{0}<\infty$. Then $\left(u_{i}^{(1)}, c^{(1)}\right)=\left(u_{i}^{(2)}, c^{(2)}\right)$.

\section{Anti-plane shear solutions}

In this Section we study a particular sub-class of problems of the general problem discussed earlier, namely anti-plane shear problems.

When we assume that $c^{\partial}=1$ for every point at the boundary, and we define the function $d$ through

$$
c\left(x_{1}, x_{2}, t\right)=1-d\left(x_{1}, x_{2}, t\right)
$$

then $d\left(x_{1}, x_{2}, t\right)$ is a function which satisfies the same equations as $c\left(x_{1}, x_{2}, t\right)$, but with null boundary conditions. We can see that

$$
F(t) \leqslant F(0) \exp \left(-2 d_{0} \lambda_{1} t\right)
$$

where $\lambda_{1}$ is the first eigenvalue for the Laplacian operator in the region $S$ and

$$
F(t)=\frac{1}{2} \int_{S} d^{2}\left(x_{\alpha}, t\right) d a .
$$

The estimate (4.2) is a time decay estimate for the difference between the concentration and the concentration assumed on the boundary. Thus, it says that the concentration in the domain $S$ tends to the concentration at the boundary. It also gives a continuous dependence result on the initial data for the solution.

Now, we look for a continuous dependence result with respect to the displacement. We assume the existence of a positive constant $C^{*}$ (which could depend on the initial data and boundary conditions) such that

$$
\left|\dot{c}\left(x_{\alpha}, t\right)\right| \leqslant C^{*},\left(x_{1}, x_{2}\right) \in \partial S, 0<t \leqslant t_{0}<\infty .
$$

We note that the maximum principle guarantees the existence of $C^{*}$ in the particular case that the diffusivity $D$ does not depend on $\nabla u$.

Let us consider homogeneous boundary conditions for $u$, and define the function

$$
G(t)=\frac{1}{2} \int_{S}\left(\rho|\dot{u}|^{2}+\left(\mu_{0}-\mu_{1}\left(c\left(x_{1}, x_{2}, t\right)\right)|\nabla u|^{2}\right) d a .\right.
$$

We have

$$
\dot{G}(t)=-\mu_{1} \int_{S} \dot{c}\left(x_{\alpha}, t\right)|\nabla u|^{2} d a \leqslant \mu_{1} C^{*} \int_{S}|\nabla u|^{2} d a .
$$

It is worth noting that when $\mu=\mu_{0} \exp \left(-\gamma_{1} c\right)$, we can define

$$
G(t)=\frac{1}{2} \int_{R}\left(\rho|\dot{u}|^{2}+\mu(c)|\nabla u|^{2}\right) d a .
$$

We then obtain that

$$
\dot{G}(t)=-\gamma_{1} \int_{S} \dot{c}\left(x_{\alpha}, t\right) \mu|\nabla u|^{2} d a \leqslant \gamma_{1} C^{*} \int_{S} \mu|\nabla u|^{2} d a
$$


It is clear that we can obtain a positive constant $D^{*}$ such that

$$
\dot{G}(t) \leqslant D^{*} G(t)
$$

A quadrature yields

$$
G(t) \leqslant G(0) \exp \left(D^{*} t\right)
$$

which gives a bound for the solutions in terms of the initial conditions.

However, in the case when $D$ does not depend on $\nabla u$, the problem becomes easier and we can apply several alternative arguments. For instance, the equation for the concentration is the well known linear diffusion equation which can be solved directly. Thus, with the known solutions for $c$, we can introduce them into the equations of motion and solve the time dependent wave equation. It is also worth noting that in this case it is also easy to obtain the impossibility of localization of solutions.

\section{Quasi-static deformations}

In this section we consider the problem when the deformations are so slow that we can neglect the acceleration of the body.

The first thing we want to pay attention to is the fact that the uniqueness of solutions fails when $c(\mathbf{x}) \equiv 1, \lambda_{0}=\lambda_{1}$ and $\mu_{0}=\mu_{1}$. This is an obvious consequence of the physics of the problem for in this case one can engender infinite strains by the application of finite stress. If we look for solutions of the form

$$
u_{i}(\mathbf{x}, t)=f^{*}(t) u_{i}^{(0)}(\mathbf{x})
$$

where $f^{*}(t)$ is an arbitrary function of time and $u_{i, i}^{(0)}=0$, we obtain that the pair $\left(u_{i}, c\right)$ satisfies our system of equations. To be precise we should set down the boundary value problem for the displacement. It is clear that if we assume the $u_{i}$ vanishes on the boundary and $u_{i}^{(0)}(\mathbf{x})$ is an arbitrary solution to the system of equations, then the functions of the form $f^{*}(t) u_{i}^{(0)}(\mathbf{x})$ also satisfy the same boundary value problem. Thus, we see that the uniqueness of solution does not hold in this case. When we assume exponential type dependence for the Lamé constants, the proposed example does not work and the non-uniqueness of a solution cannot be proved using such a methodology.

Now, we consider the case of anti-plane deformations and the case $\mu_{0}>\mu_{1}$. We want to investigate the uniqueness of solutions. As for the dynamical case, we assume the existence of two solutions $\left(u^{(j)}, c^{(j)}\right), j=1,2$ such that they satisfy $(2.14)_{2}-(2.17)$ and such that $c^{(j)}$ satisfies $0<c^{(j)} \leqslant 1$ for $j=1,2$ and

$$
u^{(j)} \in C^{2,0} \cap C^{0,1}, c^{(j)} \in C^{0,1} .
$$

Then, $u_{, k l}^{(j)}, \dot{u}^{(j)}$ and $\dot{c}^{(j)}$ are bounded on $B$, for every $0 \leqslant t \leqslant t_{0}<\infty$.

We define the functions

$$
w=u^{(1)}-u^{(2)}, h=c^{(1)}-c^{(2)} .
$$

The functions $(w, h)$ satisfy the problem determined by the system

$$
\begin{gathered}
0=\left(\mu\left(c^{(1)}\right) w_{, j}\right)_{, j}-\left(\mu_{1} h u_{, j}^{(2)}\right)_{, j} \\
h_{, t}=\left(D\left(\varepsilon_{k l}^{(1)}\right) c_{, i}^{(1)}\right)_{, i}-\left(D\left(\varepsilon_{k l}^{(2)}\right) c_{, i}^{(2)}\right)_{, i}
\end{gathered}
$$


the initial conditions

$$
h(\mathbf{x}, 0)=0 \quad \mathbf{x} \in B
$$

and the boundary conditions

$$
w(\mathbf{x}, t)=h(\mathbf{x}, t)=0 \quad(\mathbf{x}, t) \in \partial B \times\left(0, t_{0}\right) .
$$

If we define the function

$$
E(t)=\frac{1}{2} \int_{S} h^{2} d a
$$

we have

$$
\frac{d E}{d t}=-\int_{S} D\left(\nabla u^{(1)}\right) h_{, i} h_{, i} d a-\int_{B}\left(D\left(\nabla u^{(1)}\right)-D\left(\nabla u^{(2)}\right)\right) h_{, i} c_{, i}^{(2)} d v .
$$

In view of the assumptions on the function $D$, we can guarantee that

$$
\frac{d E}{d t} \leqslant K_{1} \int_{S} \mu\left(c^{(1)}\right) w_{, j} w_{, j} d a+\delta \int_{S} D h_{, j} h_{, j} d a-\int_{S} D h_{, i} h_{, i} d a .
$$

Here $\delta$ is a positive constant that we can pick as small as we want. We also can see that

$$
\int_{S} \mu\left(c^{(1)}\right) w_{, j} w, j d s=\int_{S} \mu_{1} u_{, j}^{(2)} h w, j d a .
$$

In view of this equality we can obtain the existence of a positive constant $K_{2}$ such that

$$
\int_{S} \mu\left(c^{(1)}\right) w, j w, j d s \leqslant K_{2} \int_{S} h^{2} d a
$$

It follows that there exists a constant $K_{3}$ such that

$$
\frac{d E}{d t} \leqslant K_{3} E(t)
$$

After a quadrature and on using null initial conditions, we can conclude that $h(t)=0$ for every $t \in\left(0, t_{0}\right)$. Then $w$ satisfies $0=\left(\mu\left(c^{(1)}\right) w_{, j}\right)$, which in view of the boundary conditions allow us to conclude that $w$ also vanishes and the uniqueness of solution follows. Thus, we have proved:

THEOREM 5.1 . Let $\left(u^{(j)}, c^{(j)}\right), j=1,2$ be two solutions satisfying condition (6.2) of the problem determined by the system (2.11)-(2.12), when we assume the conditions (2.5), (2.6), (2.7), (2.8), the boundary conditions $(2.16)$ and the initial conditions $(2.15)_{3}$ for every $t \in\left(0, t_{0}\right)$ where $t_{0}<\infty$. Then $\left(u^{(1)}, c^{(1)}\right)=\left(u^{(2)}, c^{(2)}\right)$.

The arguments that we have used here can be adapted to obtain a continuous dependence result on the initial data and supply terms for the system corresponding to the quasi-static and incompressible case. We first note that the function $d=1-c$ satisfies

$$
d_{t}+\left(d \dot{u}_{i}\right)_{, i}=\left(D\left(\varepsilon_{k l}\right) d_{, i}\right)_{, i}-g(\mathbf{x}, t) .
$$

Let us consider

$$
E(t)=\frac{1}{2} \int_{B} d^{2} d v
$$


We have

$$
\frac{d E}{d t}=-\int_{B} D d_{, i} d_{, i} d v-\frac{1}{2} \int_{B} d^{2} \dot{u}_{i, i} d v-\int_{B} d g d v
$$

Poincaré's inequality implies the following estimate

$$
\frac{d E}{d t} \leqslant-\int_{B} D d_{, i} d_{, i} d v+2\left(\int_{B} g^{2} d V\right)^{1 / 2} E(t)^{1 / 2} .
$$

Thus, we see that

$$
E(t) \leqslant E(0)+2 \int_{0}^{t} h_{*}(\tau)^{1 / 2} E(\tau)^{1 / 2} d \tau
$$

where

$$
h_{*}(t)=\int_{B} g^{2}(\mathbf{x}, t) d v
$$

From the Grownwall inequality we obtain that

$$
E(t)^{1 / 2} \leqslant E(0)^{1 / 2}+\int_{0}^{t} h_{*}(s) d s .
$$

We can also obtain the following estimate:

$$
\int_{B} \mu u_{i, j} u_{i, j} d v=\int_{B} f_{i} u_{i} d v .
$$

In view of the Poincaré inequality we can obtain that

$$
\int_{B} \mu u_{i, j} u_{i, j} d v \leqslant K_{2} \int_{B} f_{i} f_{i} d v
$$

where $K_{2}$ can be easily calculated. Estimates (5.20) and (5.22) imply continuous dependence of the solution on initial data and supply terms.

\section{Conclusion}

In this paper we have analyzed the system of equations that governs the degradation of a linearized elastic solid due to the diffusion of a fluid. We have established that:

1. Under suitable conditions (see (2.5)-(2.9)) we can obtain the uniqueness of solution.

2. Bounds exist for anti-plane shear deformations.

3. In the case of quasi-static deformations except for a very special condition concerning the material parameters and when $c=1$, the anti-plane shear problem has uniqueness of solutions.

\section{Acknowledgment}

The work of K.R.R. is supported by the National Science Foundation. This work is part of the project "Análisis Matemático de las Ecuaciones en Derivadas Parciales de la Termomecánica" submitted to the Spanish Ministry of Economy and Competitiveness. 


\section{REFERENCES}

AtKin, R. J. \& CRAINE, R. E. (1976) Continuum theories of mixtures: basic theory and historical development. Quat. J. Mech. Appl. Math., 29, 209-243.

Bowen, R. M. (1976) Theory of Mixtures, in Continuum Physics III (Ed. A. C. Eringen), Academic Press, New York 689-722.

BouAdi, H. \& Sun, C. T. (1989) Hygrothermal effects on the stress field of laminated composites, Journal of Reinforced Plastics and Composites, 8, 40-54.

BouAdi, H. \& Sun, C. T. (1990) Hygrothermal effects on the strucutural stiffness and strucutural damping of composites, Journal of material Science, 25, 499-505.

DARbHA, S. \& RAJAgopal, K. R. (2009) Unsteady motions of a degrading or aging linearized elastic solid, International Journal Non-Linear Mechanics, 44, 478-485.

DARCY, H. (1856) Les fontaines publiques de la ville de Dijon Victor Dalmont.

FICK, A. (1855) Über diffusion, Ann. Phys., 94, 59-86.

Kardomateas, G. A. \& Chung, C. B. (1993) Bucking of a thick ortotropic cylinder shell under external pressure including hygroscopic effects, AMD, Mechanics of Thick Composites, ASME, 213-226.

Muliana, A. Shankar, S. \& Rajagopal, K. R. (2009) Degradation of an elastic composite cylinder due to the diffusion of a fluid, Journal of Composite, 43, 1225-1249.

Rajagopal, K. R. \& TAO, L.(1995) Mechanics of Mixtures World Scientific, Singapore.

Rajagopal, K. R. \& Wineman, A. (2004) A note on viscoelastic materials that can age, International Journal of Non-Linear Mechanics, 39, 1547-1554.

SAMOHYL, I. (1987) Thermodynamics of Irreversible processes in Fluid Mixtures, Teubner, Leipzig.

Snead, J. M. \& Polazotto, A. N. (1983) Moisture and temperature effects on the instability of cylindrical composite panels, Journal of Aircraft, 20, 773-783.

SoAres, J . Moore, J. \& RAJAgopal, K. R.(2010) Deformation-induced hydrolysis of a degradable polymeric cylindrical annulus, Biomechanical Modeling and Mechanobiology, 9, 177-186.

Truesdell, C.(1957a) Sulla basi della thermomechanica, Rendiconti Lincei, 22, 33-38.

Truesdell, C.(1957b) Sulla basi della thermomechanica, Rendiconti Lincei, 22, 158-166.

TRuesdell, C. (1984) Thermodynamics, Springer-Verlag.

Weistman, Y. (1987a) Stress assisted diffusion in elastic and viscoelastic materials, Journal of Mechanics and Physics of Solids, 35, 73-93.

Weistman, Y. (1987b) Coupled damage and moisture transport in fiber reinforced, polymer composites, International Journal of Solids and Structures, 23, 1003-1025.

YEN, S. Y. \& YEN, W. J.(1989) Hygrothermal effects on the stability of cylindrical composite shell panel,Computers and Structure, 33, 551-559. 